\title{
The impact of assistants' reverse alignment surgical skill proficiency on laparoscopic colorectal surgery
}

Seunghun Lee

Department of Surgery, Kosin University Gospel Hospital, Kosin University College of Medicine, Busan, Korea

\section{Graphic Abstract}

\section{Purpose To investigate the difference in the surgical outcome based on whether the assistant overcame the mirror image in laparoscopic colorectal surgery.}

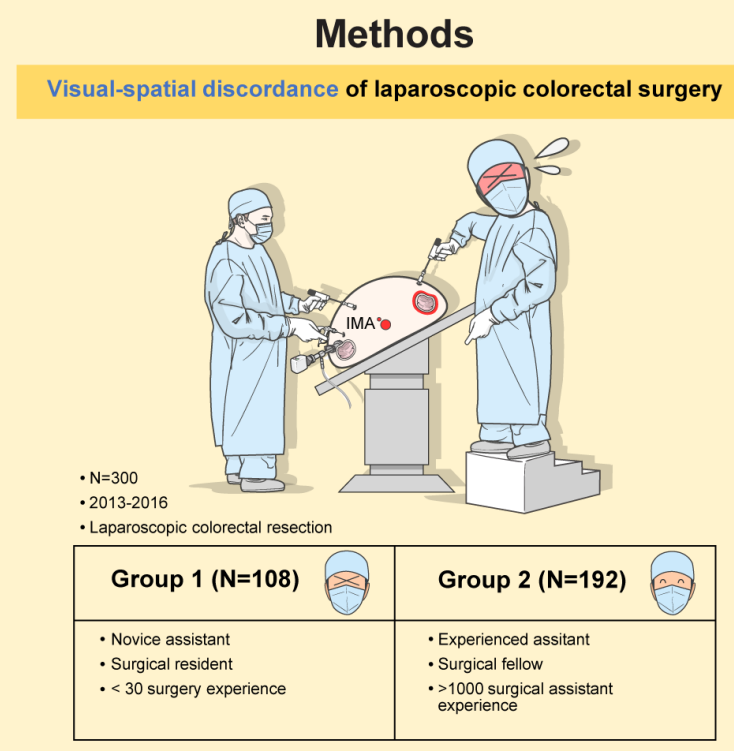

Conclusion
Assistants' surgical skill proficiency is a factor that can affect the operation time and perioperative outcomes.

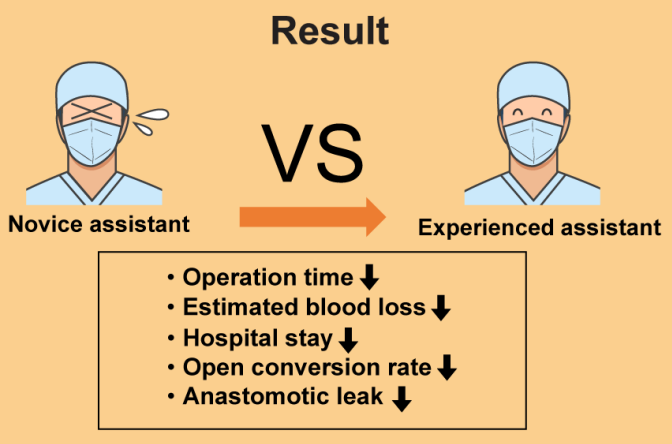

Factors related to operation time

\begin{tabular}{|l|l|}
\hline $\begin{array}{l}\text { All laparoscopic colorectal } \\
\text { resection }(n=300)\end{array}$ & Assistant ( OR =2.416) \\
\hline AR, LAR, uLAR ( $n=182)$ & $\begin{array}{l}\text { Assistant (OR=4.190) } \\
\text { BMI (OR=3.565) }\end{array}$ \\
\hline
\end{tabular}

$O R$, odds ratio; $A R$, anterior resection; $L A R$, low anterior resection; $U L A R$, ultralow anterior resection; $B M I$, body mass index
Received: Aug 1, 2021 - Revised: Sep 26, 2021 - Accepted: Oct 12, 2021 Correspondence to: Seunghun Lee, M.D., Ph.D.

Department of Surgery, Kosin University Gospel Hospital, Kosin University College of Medicine, 262 Gamcheon-ro, Seo-gu, Busan 49267, Korea

Tel: +82-51-990-6181, Fax: +82-51-246-6093

E-mail: candoli8182@naver.com

ORCID: https://orcid.org/0000-0001-9041-3156
() 2022 The Korean Society of Coloproctology

This is an open-access article distributed under the terms of the Creative Commons Attribution NonCommercial License (https://creativecommons.org/licenses/by-nc/4.0) which permits unrestricted noncommercial use, distribution, and reproduction in any medium, provided the original work is properly cited. 
Purpose: We aimed to investigate the difference in the surgical outcome based on whether the assistant overcame the mirror image in laparoscopic colorectal surgery.

Methods: Three hundred patients who underwent laparoscopic colorectal surgery performed by single operator were divided into 2 groups. Based on the assistants' experience, patients who underwent surgery involving 1 of 6 residents with an experience of fewer than 30 surgeries each were classified into group 1. Patients who underwent surgery involving a single fellow as an assistant with an experience of over 1,000 surgeries were classified into group 2 . According to the type of surgery, patients were divided into left and right colon resection groups and the surgical outcome of groups 1 and 2 was investigated.

Results: Group 2 exhibited shorter operation time, less bleeding, shorter postoperative hospital stay, lower open conversion, and anastomotic leakage rate than group 1. In right colon resection, the operation time was shorter in group 2. In left colon resection, group 2 exhibited shorter operation time, less bleeding, shorter postoperative hospital stay, and lower anastomotic leakage rate. In the multivariate analysis, the assistant was a factor affecting the operation time in the entire surgery. Conclusion: Assistants' reverse alignment surgical skill proficiency was a factor affecting the operation time.

\section{Keywords: Colorectal surgery; Laparoscopy; Learning curve}

\section{INTRODUCTION}

Laparoscopic colorectal surgery has been recognized as a standard treatment for colorectal cancer. It has become common due to similar oncologic outcome, faster recovery, and better cosmetic outcomes compared to traditional laparotomy [1-5]. However, laparoscopic colorectal surgery requires a considerable amount of surgical experience to overcome the learning curve due to the absence of tactile sense, surgery in the limited surgical field, and the use of various surgical instruments [6, 7]. Many studies have discussed the learning curve of the operator in laparoscopic colorectal surgery. It has been reported that the operator generally needs an experience of 30 to 70 surgeries to overcome the learning curve [6-8]. Several studies have evaluated the proficiency of the assistant $[9,10]$. However, comparative studies regarding the surgical outcome according to the proficiency of the assistant are insufficient.

During laparoscopic colorectal surgery, the assistant has to overcome the difficulty of laparoscopic technique and the confusion in correlating the 2-dimensional surgical field visible on the monitor and the actual 3-dimensional field in the abdominal cavity (visual-spatial discordance). Unlike the operator and the endoscopist who are positioned on the same side during laparoscopic colorectal surgery, the assistant on the opposite side encounters circumstances where the camera and working instruments are not aligned along the same axis (mirror image). A mirror image refers to an image in which the position and the direction of the surgical instruments used by the surgeon and the intraperitoneal organs of a patient are distorted in $180^{\circ}$ in upward, downward, left, and right directions on a monitor (reverse alignment condition). If the operator pushes the surgical instrument to the right and away, the assistant must pull the instrument to the left and closer to place the surgical instrument at the intended point. However, the assistant's monitor image shows the surgical instrument moving to the right and away (Fig. 1A). The assistant standing on the opposite side of the operator has no choice but to face an uncomfortable posture and unstable gripping the surgical instrument (Fig. 1B). Moreover, unlike laparoscopic surgery in other organs, in laparoscopic colorectal surgery, the surgical range is over $360^{\circ}$ from the appendix to the anorectum in the pelvic cavity. Thus, in almost all colorectal surgeries, the assistant experiences a mixture of forward and reverse alignment of images. The surgical technique to overcome this mirror image is termed reverse alignment surgical skill. It is an important ability of the assistant in laparoscopic colorectal surgery. The assistant who is not familiar with the reverse alignment surgical skill may experience confusion during surgery and affect the surgical outcome.

A few studies have reported that a surgical experience of at least 30 to 40 cases is required to overcome the mirror image in laparoscopic colorectal surgery [10]. However, no study has investigated the difference in the surgical outcome based on whether the mirror image was overcome. A difference in the surgical outcome according to the proficiency of the assistant in surgery performed by the same operator implies that efforts will be needed to find a method for improving the reverse alignment surgical skill.

The aim of the present study was to investigate the difference in the surgical outcome according to the assistant's reverse alignment surgical skill in laparoscopic colorectal surgery performed by single operator.

\section{METHODS}

From January 2013 to February 2016, consecutive 300 patients with primary adenocarcinoma of the appendix and colorectum 


\section{Coloproctology sengliun lee}
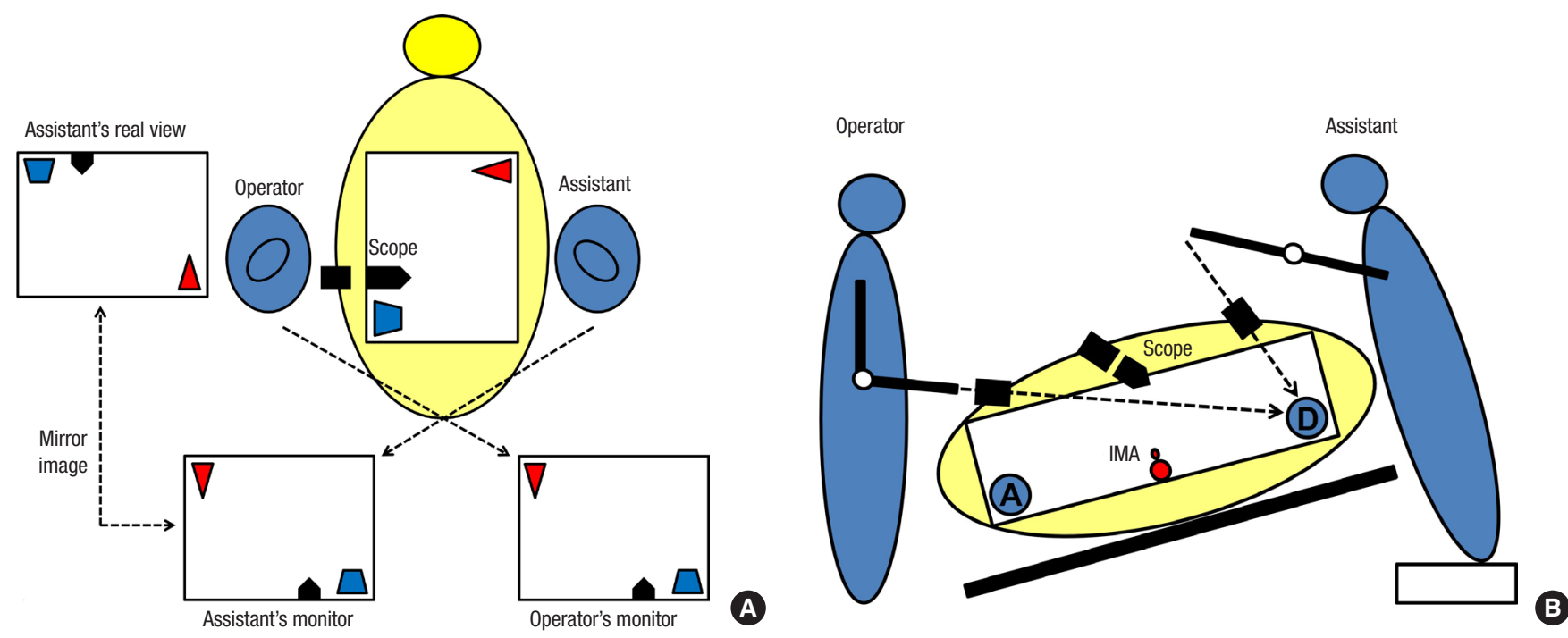

Fig. 1. Mirror image in laparoscopic colorectal surgery. (A) Coronal image. (B) Axial image (A, ascending colon; D, descending colon; IMA, inferior mesenteric artery).

who underwent laparoscopic colorectal resection performed by single operator who overcame the learning curve were included (Fig. 2). Three categories were investigated, patient factor, tumor factor, and surgical factor. According to the degree of experience in laparoscopic colorectal surgery, the assistants were divided into 2 groups. Patients' age, sex, height, weight, body mass index, medical history (hypertension, diabetes mellitus, pulmonary tuberculosis, and hepatitis), comorbidities, American Society of Anesthesiologists (ASA) physical status classification, and abdominal surgical history were investigated. Among tumor factors, information about tumor location, distance of the tumor from the anus, American Joint Committee on Cancer TNM stage (depth of invasion, nodal status, and distant metastasis), lymphovascular invasion, perineural invasion, neoadjuvant chemotherapy, obstruction, and perforation was collected. The surgical factors included the type of surgery (segmental resection, right hemicolectomy, left hemicolectomy, anterior resection, low anterior resection, ultra-low anterior resection, or abdominoperineal resection), operation time, estimated blood loss, open conversion rate, number of harvested lymph nodes, anastomosis (method and distance from the anus), anastomotic leakage rate, and postoperative hospital stay.

This study was performed in accordance with the Declaration of Helsinki. Written informed consent was obtained from all patients for the use of their clinical data in the future. The present study was approved by the Institutional Review Board of the Kosin University Gospel Hospital in Busan, Korea (No. 2020-05-027).

Five trocars were used. The operator and the endoscopist were positioned on the right side of the patient in left colorectal resection and on the left side of the patient in right hemicolectomy. The assistant was on the opposite side of the operator. The moni- tors were placed on both sides of the patient's head in right and left hemicolectomy and on both sides of the patient's leg in anterior resection, low anterior resection, ultra-low abdominal resection, and abdominoperineal resection. Surgery was performed according to the standardized procedure for laparoscopic colorectal resection. Mobilization and feeding vessel ligation with D2 lymph node dissection were performed using the medial to lateral approach in left colon resection and the lateral to medial approach in right colon resection. For ileocolostomy and colocolostomy, 2 linear staplers were used to perform extracorporeal functional end-to-end anastomosis. For anterior resection, low anterior resection, and ultra-low anterior resection, intracorporeal end-toend anastomosis was performed with double stapling using an endoscopic linear stapler and a circular stapler.

For lateral to medial access in right colon resection, the operator incises the lateral attachment of the right colon, the assistant holds the appendix or cecum with the right grasper, and the left grasper holds the mesentery of the distal ileum close to superior mesenteric vessel and pulls them in the cephalic and medial directions. The mobilization of the right colon is continued by the operator until the second and third portion of duodenum, head of pancreas, bifurcation of the ileocolic vessels from superior mesenteric vessels, and the gastrocolic trunk of Henle are exposed, and the assistant controls the degree and direction of traction of the right colon mesentery. The operator divides the avascular plane between the mesocolon and the retroperitoneum. The assistant holds the mesentery of the ileocolic vessels with the right grasper and middle colic vessels with the left grasper and pulls ileocolic vessels right laterally and middle colic vessels in the cephalic directions for vessel ligation. The assistant holds the omentum like a curtain as the operator separates the omentum from the colon and approaches the 


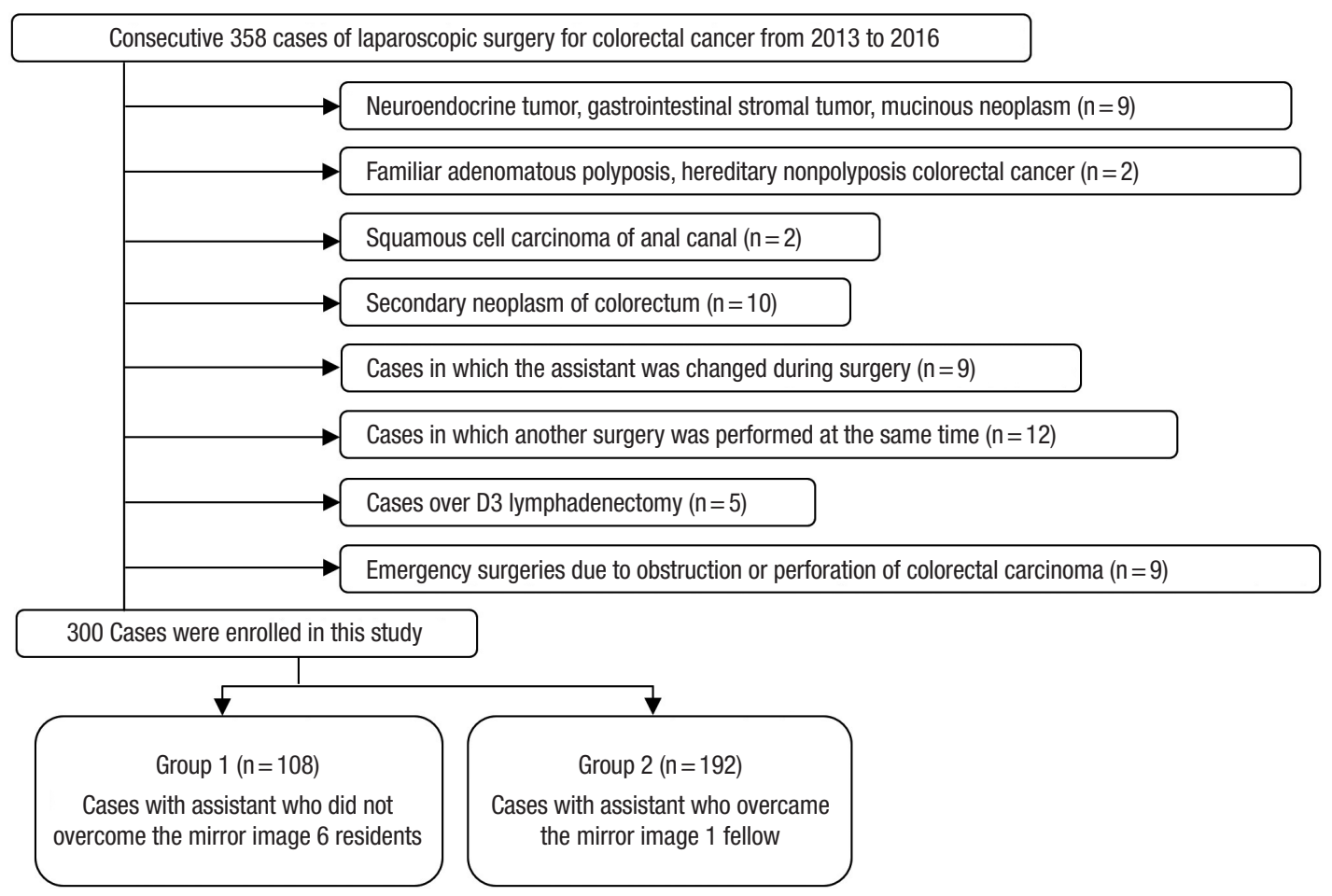

Fig. 2. Exclusion criteria for patients.

lesser sac. As the assistant lifts the stomach in ventral directions with the left grasper and pulls the right colon in the caudal directions with the right grasper, the operator incises the hepatocolic ligament to expose the pylorus and first portion of duodenum.

For medial to lateral dissection in left colon resection, while assistant holds the sigmoid colon mesentery with the left grasper, and the mesentery of the inferior mesenteric artery or vein with the right grasper and pulls them in the ventral and left lateral directions, the operator opens the pelvic peritoneum at the level of the sacral promontory. The operator divides the avascular plane between the left colon mesentery and the retroperitoneum until inferior mesenteric vessels are isolated. In this process, the assistant lifts the left colon mesentery while changing the grip point of the grasper so that the left colon mesentery can be easily separated from the retroperitoneum. After ligation of inferior mesenteric vessels, the assistant lifts the left colon mesentery a little further in the ventral and left lateral directions to help identify the ureter and gonadal vessels and divide the Toldt's fascia and facilitates medial to lateral dissection to the pancreas, Gerota's fascia, and left iliopsoas fossa. While dissection of the lateral attachment of the left colon, the assistant pulls the left colon mesentery medially with the right grasper and pulls the left parietal peritoneum laterally with the left grasper. For splenic flexure mobilization, the assistant holds the omentum like a curtain as the operator separates the omentum from the colon and accesses the lesser sac. As the operator incises the splenocolic ligament to expose the pancreas, the assistant pulls the left colon in the medial and caudal directions with both graspers. During posterior pelvic approach, the assistant holds both edge of visceral peritoneum of upper rectum and pulls them in the cephalic and ventral directions to create presacral space. The assistant lifts the rectum while changing the grip point of the graspers deeper so that the operator can dissect the presacral areola and access the pelvic floor through a Waldeyer's fascial incision.

For right lateral pelvic approach, the assistant holds the right visceral peritoneum of upper rectum with the right grasper and pulls it in the cephalic and left lateral directions and pushes the right pelvic wall right laterally with the left grasper and the operator divides the right lateral ligament of rectum. For left lateral pelvic approach, the assistant holds the left visceral peritoneum of upper rectum with the right grasper and pulls it in the cephalic and right lateral directions and pushes the left pelvic wall left laterally with the left grasper. For anterior pelvic approach, the assistant lifts the peritoneal reflection with the left grasper and pulls rectum in the cephalic and dorsal directions with the right grasper. During deep anterior dissection, the assistant lifts the seminal vesicle or vagina for dissection of the Denonvillier's fascia or rectovaginal septum. In pelvic access, the assistant facilitates the operator to identify the correct anatomical plane, preserve the hypogastric nerves and pelvic plexus, and avoid the presacral bleeding and injury of the seminal vesicle or vagina.

Altogether, 300 patients were classified into 2 groups. Based on 
assistants' experience, patients who underwent surgery involving one of 6 residents with an experience of fewer than 30 surgeries each were classified into group 1 . Patients who underwent surgery involving a single fellow as an assistant with an experience of over 1,000 surgeries were classified into group 2 . The patients were divided into the right colon resection group and the left colon resection group according to the type of surgery. In each of the groups, short-term postoperative outcome determined by the operation time, estimated blood loss, open conversion rate, number of harvested lymph nodes, anastomotic leakage rate, and the postoperative hospital stay was compared.

For statistical processing, PASW Statistics for Windows ver. 18.0 (IBM Corp., Armonk, NY, USA) was used. The independent Student $\mathrm{t}$-test and Mann-Whitney test were used to compare continuous variables and mean values. Cross-analysis was used for noncontinuous variables, odds ratios, and the univariate analysis. Logistic regression was used to analyze the hazard ratio of operation time and the multivariate analysis. Data were validated using the chi-square test and Fisher exact test. A P-value of $<0.05$ was considered statistically significant in all analyses.

\section{RESULTS}

Among all the surgeries performed concurrently, 1 of 6 residents participated as assistant in a random order in a series of 108 cases and a full-time fellow participated consecutively in 192 cases as an assistant. No significant differences were observed in patient factors, tumor factors, and the types of surgery between the groups (Table 1). Regarding surgical outcome, group 2 exhibited shorter operation time, less bleeding, shorter postoperative hospital stay,

Table 1. Demographics, tumor characteristics, and surgical data

\begin{tabular}{lccc}
\hline Variable & Group 1 & Group 2 & P-value \\
\hline No. of patients & 108 & 192 & \\
Sex & & & 0.23 \\
$\quad$ Male & $66(61.1)$ & $103(53.6)$ & \\
$\quad$ Female & $42(38.9)$ & $89(46.4)$ & \\
Age (yr) & & & 0.31 \\
$\quad \leq 60$ & $42(38.9)$ & $63(32.8)$ & \\
$>60$ & $66(61.1)$ & $129(67.2)$ & \\
Body mass inces $\left(\mathrm{kg} / \mathrm{m}^{2}\right)$ & & & 0.60 \\
$\quad \leq 25$ & $78(72.2)$ & $132(68.8)$ & \\
$>25$ & $30(27.8)$ & $60(31.3)$ & \\
ASA PS classification & & & 0.55 \\
I & $10(9.3)$ & $18(9.4)$ & \\
॥I & $79(73.1)$ & $149(77.6)$ & \\
III & $19(17.6)$ & $25(13.0)$ & \\
\hline
\end{tabular}

(Continued to the next)
Table 1. Continued

\begin{tabular}{|c|c|c|c|}
\hline Variable & Group 1 & Group 2 & P-value \\
\hline Previous laparotomy & & & 0.75 \\
\hline Yes & $17(15.7)$ & $34(17.7)$ & \\
\hline No & $91(84.3)$ & $158(82.3)$ & \\
\hline Tumor location & & & 0.10 \\
\hline Appendix & $1(0.9)$ & $0(0)$ & \\
\hline Cecum & $2(1.9)$ & $0(0)$ & \\
\hline Ascending colon & $21(19.4)$ & $31(16.1)$ & \\
\hline Hepatic flexure & $5(4.6)$ & $10(5.2)$ & \\
\hline Transverse colon & $4(3.7)$ & $15(7.8)$ & \\
\hline Splenic flexure & $4(3.7)$ & $4(2.1)$ & \\
\hline Descending colon & $3(2.8)$ & $6(3.1)$ & \\
\hline Sigmoid colon & $31(28.7)$ & 35 (18.2) & \\
\hline Rectosigmoid junction & $17(15.7)$ & $45(23.4)$ & \\
\hline Rectum & 20 (18.5) & $46(24.0)$ & \\
\hline Preoperative CCRT & & & 0.73 \\
\hline Yes & $4(3.7)$ & $5(2.6)$ & \\
\hline No & 104 (96.3) & $187(97.4)$ & \\
\hline Obstruction & & & 0.16 \\
\hline Yes & 24 (22.2) & $30(15.6)$ & \\
\hline No & $84(77.8)$ & $162(84.4)$ & \\
\hline Perforation & & & 0.36 \\
\hline Yes & $3(2.8)$ & $2(1.0)$ & \\
\hline No & $105(97.2)$ & $190(99.0)$ & \\
\hline Stage & & & 0.46 \\
\hline 0 & $1(0.9)$ & $3(1.6)$ & \\
\hline I & $27(25.0)$ & $57(29.7)$ & \\
\hline$\|$ & $36(33.4)$ & $68(35.4)$ & \\
\hline III & 37 (34.3) & $59(30.8)$ & \\
\hline IV & $7(6.5)$ & $5(2.6)$ & \\
\hline Surgery & & & 0.05 \\
\hline Segmental resection & $4(3.7)$ & $13(6.8)$ & \\
\hline Right hemicolectomy & $30(27.8)$ & $49(25.5)$ & \\
\hline Left hemicolectomy & $9(8.3)$ & $8(4.2)$ & \\
\hline Anterior resection & $33(30.6)$ & $38(19.8)$ & \\
\hline Low anterior resection & 23 (21.3) & $68(35.4)$ & \\
\hline Ultra-low anterior resection & $7(6.5)$ & $13(6.8)$ & \\
\hline Abdominoperineal resection & $2(1.9)$ & $3(1.6)$ & \\
\hline
\end{tabular}

Values are presented as number only or number (\%).

Group 1, patients who underwent surgery involving 1 of 6 residents with an experience of fewer than 30 surgeries each; group 2, patients who underwent surgery involving a single fellow as an assistant with an experience of over 1,000 surgeries. ASA, American Society of Anesthesiologists; PS, physical status; CCRT, concurrent chemoradiation therapy. 
lower open conversion rate, and lower anastomotic leakage rate (Table 2). In right colon resection, the operation time was significantly shorter in group 2 (Table 3 ). In left colon resection including anterior resection, low anterior resection, and ultra-low anterior resection, group 2 exhibited shorter operation time, less bleeding, shorter postoperative hospital stay, and lower anastomotic leakage rate (Table 3). In the univariate analysis based on average operation time of 165.3 minutes, the assistant was factor affecting the operation time (Table 4). In right colon resection, the assistant was factor affecting the operation time (mean, 166.7 minutes) (Table 5). In left colon resection, the assistant, body mass index, distance of the lesion from the anus, and the type of surgery were factors affecting the operation time (mean, 163.2 minutes) (Table 5). In the multivariate analysis, the assistant was factor affecting the operation time in the entire surgery (Table 6). No significant independent factor was found in right colon resection. The assistant and body mass index were identified as independent factors affecting the operation time in left colon resection (Table 6).

\section{DISCUSSION}

The assistant's role in laparoscopic colorectal surgery is to help the

Table 2. Surgical outcome (total)

\begin{tabular}{lccr}
\hline Total & Group 1 $(\mathrm{n}=108)$ & Group 2 $(\mathrm{n}=192)$ & P-value \\
\hline Operation time (min) & $197.5 \pm 70.4$ & $147.2 \pm 38.8$ & $<0.05$ \\
Estimated blood loss (mL) & $88.4 \pm 131.5$ & $42.6 \pm 65.4$ & $<0.05$ \\
Harvested lymph nodes & $18.5 \pm 6.5$ & $18.9 \pm 4.9$ & 0.64 \\
Postoperative hospital stay (day) & $9.0 \pm 4.9$ & $7.9 \pm 2.3$ & 0.01 \\
Open conversion & $4(3.7)$ & $0(0)$ & 0.02 \\
Anastomotic leakage & $3(2.8)$ & $0(0)$ & 0.04 \\
\hline
\end{tabular}

Values are presented as mean \pm standard deviation and number (\%).

Group 1, patients who underwent surgery involving 1 of 6 residents with an experience of fewer than 30 surgeries each; group 2, patients who underwent surgery involving a single fellow as an assistant with an experience of over 1,000 surgeries. operator in the surgical procedure by lifting, pulling, and pushing tissue. In laparoscopic colorectal surgery, the role of the assistant ranges from the passive role of holding the tissue designated by the operator to the active role of understanding the surgical process, anticipating the next process, holding the necessary tissue by itself, and performing counter-traction according to the dissection plane. Unlike traditional open colorectal surgery, role of the assistant in laparoscopic surgery is greatly affected by the reverse alignment surgical skill. In laparoscopic colorectal surgery, the assistant's clumsy reverse alignment surgical skill simply means that 'I cannot grab what you and I want.' In laparoscopic colorectal surgery, the proportion of extracorporeal procedures such as extracorporeal anastomosis and circular stapler insertion through the anus is small, and the role of assistants in extracorporeal procedures is very limited. Therefore, it is safe to say that the proficiency of the assistant in laparoscopic colorectal surgery is synonymous with the proficiency of the reverse alignment surgical skill. Usually, the learning curve for laparoscopic surgery is analyzed in terms of surgical outcome determined by operation time, estimated blood loss, open conversion rate, number of harvested lymph nodes, anastomotic leakage rate, and postoperative hospital stay [7]. Some reports have suggested that experience of the operator has a great influence on the outcome of laparoscopic colorectal surgery and the operator must have an experience of dozens of surgeries to overcome the learning curve [6-8]. However, the relationship between the proficiency of the operator and the surgical outcome can be easily understood even without statistical analysis. Very few studies have analyzed assistants' proficiency including the reverse alignment surgical skill, which is essential for overcoming visual-spatial discordance, a special situation faced by the assistant in laparoscopic colorectal surgery $[9,10]$. Moreover, no clinical studies have analyzed the effect of assistants on surgical outcome. A study published in 2010 concluded that an experience of 30 to 40 surgical cases was necessary for the assistant to overcome the learning curve [10]. The study measured the execution time and the error rate for grasping tissue through video review. However,

Table 3. Surgical outcome (type of surgery)

\begin{tabular}{|c|c|c|c|c|c|c|}
\hline \multirow{2}{*}{ Type of surgery } & \multicolumn{3}{|c|}{$\operatorname{RHC}(n=79)$} & \multicolumn{3}{|c|}{ AR, LAR, uLAR $(n=182)$} \\
\hline & Group $1(n=30)$ & Group $2(n=49)$ & P-value & Group $1(n=63)$ & Group $2(n=119)$ & P-value \\
\hline Operation time (min) & $195.6 \pm 63.7$ & $149.0 \pm 32.0$ & $<0.05$ & $199.3 \pm 72.0$ & $144.1 \pm 39.0$ & $<0.05$ \\
\hline $\mathrm{EBL}(\mathrm{mL})$ & $106.6 \pm 201.5$ & $50.2 \pm 74.0$ & 0.08 & $72.8 \pm 78.8$ & $40.5 \pm 66.0$ & $<0.05$ \\
\hline Harvested LNs & $20.6 \pm 6.0$ & $19.9 \pm 5.8$ & 0.60 & $18.0 \pm 6.7$ & $18.3 \pm 4.4$ & 0.71 \\
\hline Postoperative hospital stay (day) & $7.8 \pm 2.4$ & $8.1 \pm 2.2$ & 0.64 & $9.4 \pm 4.7$ & $7.8 \pm 2.5$ & 0.01 \\
\hline Open conversion & $2(6.7)$ & $0(0)$ & 0.14 & $1(1.6)$ & $0(0)$ & 0.35 \\
\hline Anastomotic leakage & $0(0)$ & $0(0)$ & & $3(4.8)$ & $0(0)$ & 0.04 \\
\hline
\end{tabular}

Values are presented as mean \pm standard deviation and number (\%).

Group 1, patients who underwent surgery involving 1 of 6 residents with an experience of fewer than 30 surgeries each; group 2, patients who underwent surgery involving a single fellow as an assistant with an experience of over 1,000 surgeries.

EBL, estimated blood loss; LN, lymph nodes; RHC, right hemicolectomy; AR, anterior resection; LAR, low anterior resection; uLAR, ultra-LAR. 
Table 4. Univariate analysis of factors related to operation time (total)

\begin{tabular}{|c|c|c|c|}
\hline \multirow[b]{2}{*}{ Variable } & \multicolumn{2}{|c|}{ Operation time (min) } & \multirow[b]{2}{*}{ P-value } \\
\hline & $\begin{array}{c}\leq 165.3 \\
(n=193)\end{array}$ & $\begin{array}{l}>165.3 \\
(n=107)\end{array}$ & \\
\hline Assistant & & & $<0.05$ \\
\hline Group 1 & $42(38.9)$ & $66(61.6)$ & \\
\hline Group 2 & $151(78.6)$ & $41(21.4)$ & \\
\hline Age (yr) & & & 0.38 \\
\hline$\leq 60$ & $64(61.0)$ & $41(39.0)$ & \\
\hline$>60$ & $129(66.2)$ & $66(33.8)$ & \\
\hline Sex & & & 0.81 \\
\hline Male & $110(65.1)$ & $59(34.9)$ & \\
\hline Female & $83(63.4)$ & 48 (36.6) & \\
\hline Body mass index $\left(\mathrm{kg} / \mathrm{m}^{2}\right)$ & & & 0.36 \\
\hline$\leq 25$ & $139(66.2)$ & $71(33.8)$ & \\
\hline$>25$ & $54(60.0)$ & $36(40.0)$ & \\
\hline ASA PS classification & & & 0.60 \\
\hline I & $19(67.9)$ & $9(32.1)$ & \\
\hline$\|$ & $143(62.7)$ & $85(37.3)$ & \\
\hline III & $31(70.5)$ & 13 (29.5) & \\
\hline Previous laparotomy & & & 0.63 \\
\hline Yes & $31(60.8)$ & 20 (39.2) & \\
\hline No & $162(65.1)$ & $87(34.9)$ & \\
\hline Obstruction & & & 0.43 \\
\hline Yes & $32(59.3)$ & $22(40.7)$ & \\
\hline No & $161(65.4)$ & 85 (34.6) & \\
\hline Perforation & & & 0.35 \\
\hline Yes & $2(40.0)$ & $3(60.0)$ & \\
\hline No & $191(64.7)$ & $104(35.3)$ & \\
\hline Stage & & & 0.08 \\
\hline 0 & $2(50.0)$ & $2(50.0)$ & \\
\hline I & $61(72.6)$ & $23(27.4)$ & \\
\hline$\|$ & $70(67.3)$ & $34(32.7)$ & \\
\hline III & $55(57.3)$ & $41(42.7)$ & \\
\hline IV & $5(41.7)$ & $7(58.3)$ & \\
\hline Surgery & & & 0.07 \\
\hline Segmental resection & $10(58.8)$ & $7(41.2)$ & \\
\hline Right hemicolectomy & $50(63.3)$ & $29(36.7)$ & \\
\hline Left hemicolectomy & $12(70.6)$ & $5(29.4)$ & \\
\hline Anterior resection & 47 (66.2) & $24(33.8)$ & \\
\hline Low anterior resection & 65 (71.4) & 26 (28.6) & \\
\hline Ultra-low anterior resection & $8(40.0)$ & $12(60.0)$ & \\
\hline Abdominoperineal resection & $1(20.0)$ & $4(80.0)$ & \\
\hline
\end{tabular}

Values are presented as number (\%).

Group 1, patients who underwent surgery involving 1 of 6 residents with an experience of fewer than 30 surgeries each; group 2, patients who underwent surgery involving a single fellow as an assistant with an experience of over 1,000 surgeries. ASA, American Society of Anesthesiologists; PS, physical status. it is difficult to determine whether an assistant overcomes the learning curve only by evaluating the execution time and the error rate for grasping. The reverse alignment surgical skill tends to be limited to the assistant rather than the operator, so the effect of the reverse alignment surgical skill on the surgical outcome is underestimated. There is no consensus on criteria to overcome the learning curve of reverse alignment surgical skill in laparoscopic colorectal surgery. In this study, the proficiency of the reverse alignment surgical skill was determined by the number of surgeries, and the groups were divided into residents with less than 30 surgical experiences as assistant and full-time fellow with more than 1,000 surgical experiences as assistant. In addition, this study was conducted on a single operator who had more than 3,000 laparoscopic colorectal surgeries to minimize the operator factor. We hypothesized that not only the operator, but also assistant affects the surgical outcome in laparoscopic colorectal surgery. To determine the effect of assistants' proficiency on the short-term surgical outcome, the same factors assessing the learning curve of laparoscopic colorectal surgery in 2 assistant groups with distinctly different experiences were analyzed.

In terms of the operation time, estimated blood loss, postoperative hospital stay, open conversion rate, and anastomotic leakage rate, group 2 showed better results than group 1 . The operation time, estimated blood loss, and postoperative hospital stay in group 2 were lower than those in group 1 . These findings suggest that the ability of the operator as well as the ability of the assistant to overcome the mirror image can contribute to the plateau of learning curve in laparoscopic colorectal surgery. In left colon resection (anterior, low anterior, and ultra-low anterior resection), the difference in the operation time between the assistant groups was larger than that in right colon resection. Moreover, significant differences were observed in estimated blood loss, postoperative hospital stay, and anastomotic leakage rate. The proficiency of assistants' reverse alignment surgical skill showed a statistically significant correlation with the surgical outcome.

Laparoscopic colorectal surgery was first introduced by Jacobs et al. [1] in 1991. Currently, it is a standard surgical method for benign and malignant colorectal diseases. Except in the case of first-generation surgeons who started laparoscopic colorectal surgery, colorectal surgeons begin as assistants in laparoscopic colorectal surgery and overcoming visual-spatial discordance is an important task for these assistants. Several studies related to overcoming visual-spatial discordance have been reported [9, 11-14]. A study involving a video trainer reported that reverse alignment surgical skill can be improved through experience, but it is difficult to overcome through the residency training alone and training in the forward orientation does not help improve the reverse alignment surgical skill [9]. Other studies have reported the effect of laparoscopic performance according to the position of the video monitor and the laparoscopic camera [12]. Surgeon's experience is important for superior performance, and it is ideal to place the laparoscopic camera in front of the surgeon and the monitor on 
Table 5. Univariate analysis of factors related to operation time (type of surgery)

\begin{tabular}{|c|c|c|c|c|c|c|}
\hline \multirow{3}{*}{ Variable } & \multicolumn{6}{|c|}{ Operation time (min) } \\
\hline & \multicolumn{3}{|c|}{$\operatorname{RHC}(n=79)$} & \multicolumn{3}{|c|}{ AR, LAR, uLAR $(n=182)$} \\
\hline & $\leq 166.7(n=50)$ & $>166.7(n=29)$ & P-value & $\leq 163.2(n=115)$ & $>163.2(n=67)$ & P-value \\
\hline Assistant & & & $<0.05$ & & & $<0.05$ \\
\hline Group 1 & $10(33.3)$ & $20(66.7)$ & & $24(38.1)$ & $39(61.9)$ & \\
\hline Group 2 & $40(80.0)$ & $9(18.4)$ & & $91(76.5)$ & $28(23.5)$ & \\
\hline Age (yr) & & & 0.05 & & & 0.35 \\
\hline$\leq 60$ & $42(70.0)$ & $18(30.0)$ & & $48(67.6)$ & $23(32.4)$ & \\
\hline$>60$ & $8(42.1)$ & $11(57.9)$ & & $67(60.4)$ & $44(39.6)$ & \\
\hline Sex & & & 0.64 & & & 0.53 \\
\hline Male & $22(66.7)$ & $11(33.3)$ & & $69(61.1)$ & $44(38.9)$ & \\
\hline Female & $28(60.9)$ & $18(39.1)$ & & $46(66.7)$ & $23(33.3)$ & \\
\hline Body mass index $\left(\mathrm{kg} / \mathrm{m}^{2}\right)$ & & & 1.00 & & & 0.02 \\
\hline$\leq 25$ & $36(63.2)$ & $21(36.8)$ & & $90(68.7)$ & $41(31.3)$ & \\
\hline$>25$ & $14(63.6)$ & $8(36.4)$ & & $25(49.0)$ & $26(51.0)$ & \\
\hline ASA PS classification & & & 0.12 & & & 0.27 \\
\hline I & $1(25.0)$ & $3(75.0)$ & & $16(80.0)$ & $4(20.0)$ & \\
\hline$\|$ & $35(61.4)$ & $22(38.6)$ & & $85(61.6)$ & $53(38.4)$ & \\
\hline III & $14(77.8)$ & 4 (22.2) & & $14(58.3)$ & $10(41.7)$ & \\
\hline Previous laparotomy & & & 0.79 & & & 0.83 \\
\hline Yes & $12(60.0)$ & $8(40.0)$ & & 16 (61.5) & 10 (38.5) & \\
\hline No & $38(64.4)$ & $21(35.6)$ & & 99 (63.5) & $57(36.5)$ & \\
\hline Preoperative CCRT & & & & & & 0.73 \\
\hline Yes & & & & $5(55.6)$ & $4(44.4)$ & \\
\hline No & & & & 110 (63.6) & $63(36.4)$ & \\
\hline Tumor level (cm) & & & & & & 0.03 \\
\hline$\leq 5$ & & & & 4 (33.3) & $8(66.7)$ & \\
\hline $6-10$ & & & & 19 (54.3) & $16(45.7)$ & \\
\hline$\geq 11$ & & & & $92(68.1)$ & $43(31.9)$ & \\
\hline Obstruction & & & 0.78 & & & 0.17 \\
\hline Yes & $10(58.8)$ & 7 (41.2) & & $18(52.9)$ & $16(47.1)$ & \\
\hline No & $40(64.5)$ & 22 (35.5) & & 97 (65.5) & $51(34.5)$ & \\
\hline Perforation & & & 1.00 & & & 0.14 \\
\hline Yes & $1(100)$ & $0(0)$ & & $1(25.0)$ & $3(75.0)$ & \\
\hline No & 49 (62.8) & 29 (37.2) & & $114(64.0)$ & $64(36.0)$ & \\
\hline Stage & & & 0.63 & & & 0.50 \\
\hline 0 & 1 (33.3) & $2(66.7)$ & & $1(100)$ & $0(0)$ & \\
\hline I & $12(75.0)$ & $4(25.0)$ & & $42(70.0)$ & $18(30.0)$ & \\
\hline$\|$ & $22(64.7)$ & 12 (35.3) & & $32(64.0)$ & $18(36.0)$ & \\
\hline III & 13 (56.5) & $10(43.5)$ & & 37 (56.9) & $28(43.1)$ & \\
\hline IV & 2 (66.7) & 1 (33.3) & & $3(50.0)$ & $3(50.0)$ & \\
\hline Surgery & & & & & & 0.02 \\
\hline$A R$ & & & & $46(64.8)$ & 25 (35.2) & \\
\hline LAR & & & & $62(68.1)$ & $29(31.9)$ & \\
\hline uLAR & & & & 7 (35.0) & $13(65.0)$ & \\
\hline
\end{tabular}

Values are presented as number (\%).

Group 1, patients who underwent surgery involving 1 of 6 residents with an experience of fewer than 30 surgeries each; group 2, patients who underwent surgery involving a single fellow as an assistant with an experience of over 1,000 surgeries.

RHC, right hemicolectomy; AR, anterior resection; LAR, low anterior resection; ULAR, ultra-LAR; ASA, American Society of Anesthesiologists; PS, physical status; CCRT, concurrent chemoradiation therapy. 
Table 6. Multivariate analysis of factors related to operation time

\begin{tabular}{lcc}
\hline Variable & \multicolumn{1}{c}{ OR $(95 \% \mathrm{Cl})$} & P-value \\
\hline Total $(\mathrm{n}=300)$ & & \\
$\quad$ Assistant & $2.416(1.047-5.576)$ & 0.04 \\
AR, LAR, uLAR $(\mathrm{n}=182)$ & & \\
Assistant & $4.190(1.314-13.363)$ & 0.02 \\
Body mass index & $3.565(1.295-9.814)$ & 0.01 \\
\hline
\end{tabular}

$\overline{\mathrm{OR}}$, odds ratio; $\mathrm{Cl}$, confidence interval, $\mathrm{AR}$, anterior resection; LAR, low anterior resection; ULAR, ultra-LAR.

the other side. Alternatively, the camera and the monitor can be placed facing each other near the surgeon's non-dominant hand. A study published in 2012 demonstrated that training in standard forward-alignment conditions can be useful in visual-spatial discordance situations, but intentional training of reverse alignment skill in visual-spatial discordance situations can be beneficial for beginners [13]. In addition, some studies have reported that the mirror image can be overcome by rotating the screen displayed on the assistant's monitor using an $180^{\circ}$ image converter [11]. In a recent randomized controlled trial, an experiment was conducted to study whether laparoscopic technique training in reverse and side alignment conditions could improve the forward-alignment performance [14]. However, the results were inconclusive. Training through a video trainer in a visual-spatial discordance situation requires a considerable period to master the reverse alignment surgical skill. Repositioning the laparoscopic camera and the assistant's video monitor is difficult to perform in operator-oriented surgery. The method using an image converter is unlikely to be applicable, as it cannot convert the intraperitoneal scene in real time. The scene changes continuously from $0^{\circ}$ to $360^{\circ}$ and is not fixed at $0^{\circ}, 90^{\circ}, 270^{\circ}$, or $180^{\circ}$. The most desirable method to overcome the visual-spatial discordance in laparoscopic colorectal surgery is to convert the paradoxical movement due to visual illusion in reverse alignment conditions from semantic memory to procedural memory through extensive experience.

The limitation of this study is that the proficiency evaluation criteria of the reverse alignment surgical skill are based on the clear difference in the surgical experience between the 2 groups, not the experimental data through a training box such as the peg transfer task. However, there is no objective standard for overcoming the learning curve of reverse alignment surgical skill. We believe that the difference in the number of surgeries used as the classification criteria in this study can rather be a reliable for distinguishing whether the learning curve has been overcome.

In conclusion, the present study is the first clinical study regarding the effect of reverse alignment surgical skill proficiency of an assistant who experiences visual-spatial discordance during surgery on the surgical outcome. The results of the present study suggested that assistants' surgical skill proficiency is a factor that can affect the operation time. Suggesting beginners several tips for overcoming vi- sual-spatial discordance such as setting reference points and landmarks on the monitor image, grasper gripping and handling tips, direction of gaze and traction, flow of thoughts can improve reverse alignment surgical skill and shorten operation time.

\section{CONFLICT OF INTEREST}

No potential conflict of interest relevant to this article was reported.

\section{FUNDING}

None.

\section{ORCID}

Seunghun Lee, https://orcid.org/0000-0001-9041-3156

\section{ACKNOWLEDGMENTS}

The author thanks medical staff and residents of Department of Surgery, Kosin University Gospel Hospital for their participation in this study.

\section{REFERENCES}

1. Jacobs M, Verdeja JC, Goldstein HS. Minimally invasive colon resection (laparoscopic colectomy). Surg Laparosc Endosc 1991;1: 144-50.

2. Weeks JC, Nelson H, Gelber S, Sargent D, Schroeder G; Clinical Outcomes of Surgical Therapy (COST) Study Group. Short-term quality-of-life outcomes following laparoscopic-assisted colectomy vs open colectomy for colon cancer: a randomized trial. JAMA 2002;287:321-8.

3. Jayne DG, Guillou PJ, Thorpe H, Quirke P, Copeland J, Smith $\mathrm{AM}$, et al. Randomized trial of laparoscopic-assisted resection of colorectal carcinoma: 3 -year results of the UK MRC CLASICC Trial Group. J Clin Oncol 2007;25:3061-8.

4. Lim SB, Choi HS, Jeong SY, Park JG. Feasibility of laparoscopic techniques as the surgical approach of choice for primary colorectal cancer: an analysis of 570 consecutive cases. Surg Endosc 2008; 22:2588-95.

5. Lim SW, Kim YJ, Kim HR. Laparoscopic surgery for colorectal cancer in patients over 80 years of age: the morbidity outcomes. Ann Surg Treat Res 2017;92:423-8.

6. Reissman P, Cohen S, Weiss EG, Wexner SD. Laparoscopic colorectal surgery: ascending the learning curve. World J Surg 1996;20: 277-82.

7. Schlachta CM, Mamazza J, Seshadri PA, Cadeddu M, Gregoire R, Poulin EC. Defining a learning curve for laparoscopic colorectal resections. Dis Colon Rectum 2001;44:217-22.

8. Tekkis PP, Senagore AJ, Delaney CP, Fazio VW. Evaluation of the learning curve in laparoscopic colorectal surgery: comparison of 
right-sided and left-sided resections. Ann Surg 2005;242:83-91.

9. Gould JC, Frydman J. Reverse-alignment surgical skills assessment. Surg Endosc 2007;21:669-71.

10. Hwang MR, Seo GJ, Yoo SB, Park JW, Choi HS, Oh JH, et al. Learning curve of assistants in laparoscopic colorectal surgery: overcoming mirror imaging. Surg Endosc 2010;24:2575-80.

11. Johnston WK 3rd, Low RK, Das S. Image converter eliminates mirror imaging during laparoscopy. J Endourol 2003;17:327-31.

12. Haveran LA, Novitsky YW, Czerniach DR, Kaban GK, Taylor M, Gallagher-Dorval K, et al. Optimizing laparoscopic task efficien- cy: the role of camera and monitor positions. Surg Endosc 2007; 21:980-4.

13. Holznecht C, Schmidt T, Gould J. The impact of training under different visual-spatial conditions on reverse-alignment laparoscopic skills development. Surg Endosc 2012;26:120-3.

14. Khogali-Jakary N, Kanitra JJ, Haan PS, Anderson CI, Davis AT, Henry D, et al. Laparoscopic simulation in reverse and side alignment impact on forward alignment performance: a randomized controlled trial. Surg Endosc 2020;34:298-303. 